\title{
TRANSFER FUNCTION MODELING OF DAMPING MECHANISMS IN DISTRIBUTED PARAMETER MODELS
}

\author{
J.C. Slater* and D.J. Inmant \\ Mechanical Systems Lab, Department of Mechanical and Aerospace Engineering \\ State University of New York at Buffalo, Buffalo, NY 14260
}

(716) 636-2733

\section{INTRODUCTION}

This work formulates a method for the modeling of material damping characteristics in distributed parameter models which may be easily applied to models such as rod, plate and beam equations. The general linear boundary value vibration equation is modified to incorporate hysteresis effects represented by complex stiffness using the transfer function approach proposed by Golla and Hughes. The governing characteristic equations are decoupled through separation of variables yielding solutions similar to those of undamped classical theory, allowing solution of the steady state as well as transient response. Example problems and solutions are provided demonstrating the similarity of the solutions to those of the classical theories and transient responses of non-viscous systems.

\section{BACKGROUND}

Classical damping models assume a simple damping term proportional to velocity and/or strain rate such that the equations of motion decouple readily.(See for instance Reismann ${ }^{1}$ and Soedel ${ }^{2}$ ). This modeling technique is more of ten an equivalent viscous damping representation of non-viscous damping than a representation of the real structural damping ${ }^{3}$. Banks et.al. ${ }^{4}$ have shown experimentally that hysteretic effects dominate the damping mechanisms in a cantilevered composite beam. However, analysis of hysteretic models is quite difficult. Inman 5 proposed using the Golla and Hughes viscoelastic model for modeling damping in composite beams, and Slater ${ }^{6}$ extended this theory to plates. A simple method of representing damping mechanisms over a wide frequency range which can be included in the general linear boundary value vibration equation to arrive at a closed form solution for the damped response is presented in the following.

The time hysteretic stress-strain relation is given by

$$
\sigma(\mathbf{x}, t)=\varepsilon(\mathbf{x}, t) E-\int_{0}^{1} g(t-s) \varepsilon(\mathbf{x}, s) d s
$$

where $\sigma(\mathbf{x}, t)$ is the stress, $x$ is the vector of spacial coordinates, $\varepsilon(\mathbf{x}, t)$ is the strain, and the kernel $g(t-s)$ describes the hysteresis as developed by Christensen ${ }^{7}$ or Banks ${ }^{8}$, for example. The general linear boundary value vibration equation incorporating viscous and hysteretic damping may then be written as

$$
\ddot{w}(\mathbf{x}, t)+d \dot{w}(\mathbf{x}, t)+L_{2}\left[L_{3}(\mathbf{x}) w(\mathbf{x}, t)+L_{1} w(\mathbf{x}, t)\right]=f(\mathbf{x}, t)
$$

* Graduate Research Assistant, Mechanical Systems Lab, Department of Mechanical and Aerospace Engineering

†Professor and Department Chair, Mechanical Systems Lab, Department of Mechanical and Aerospace Engineering

$$
=546
$$


where $w(\mathbf{x}, t)$ is the displacement, $d$ is the viscous damping coefficient, and $f(\mathbf{x}, t)$ is the forcing function. The operators $L_{2}$ and $L_{3}$ are stiffness operators; $L_{1}$ represents the integral part of (1) and ( ) denotes partial differentiation with respect to time.

In modeling damping using finite element models, Golla and Hughes ${ }^{9}$ have shown that the variation of material damping and stiffness properties as a function of frequency (i.e. time hysteresis) can be represented by

$$
E^{*}(s)=E\left[1+\sum_{i=1}^{k} \alpha_{i} \frac{\beta_{i} s^{2}+\gamma_{i} s}{\beta_{i} s^{2}+\gamma_{i} s+\eta_{i}}\right]
$$

where $E^{*}(s)$ is the complex modulus of elasticity, $E$ is the static modulus of elasticity, and $s$ is the Laplace domain operator. The symbols $\alpha_{n}, \beta_{n}, \gamma_{n}$, and $\eta_{n}$ represent constants for curve fitting to match complex modulus data. Ideally, $k$ would be infinite in order to perfectly match the material properties over all frequencies. However, it is up to the analyst to determine the value of $k$ in order to obtain sufficient accuracy. Although this transfer function has been developed for finite element models, it is easily extendable to distributed parameter theory.

Using (3) to model the time hysteretic stress-strain effects of (1) gives

$$
\sigma(\mathrm{x}, s)=E^{*}(s) \varepsilon(\mathrm{x}, s)=E\left[1+\sum_{i=1}^{k} \alpha_{i} \frac{\beta_{i} s^{2}+\gamma_{i} s}{\beta_{i} s^{2}+\gamma_{i} s+\eta_{i}}\right] \varepsilon(s)
$$

Incorporating the GHM model into the Laplace domain representation of (2) results in

$$
\begin{gathered}
s^{2} W(\mathbf{x}, s)-s w(\mathbf{x}, 0)-w_{1}(\mathbf{x}, 0)+s d W(\mathbf{x}, s) \\
-d w(\mathbf{x}, 0)+I_{2}\left[E^{*}(s) N(\mathbf{x}) W(\mathbf{x}, s)\right]=F(\mathbf{x}, s)
\end{gathered}
$$

where the operator $I_{1}$ has been replaced by

$$
I_{1}=E \sum_{i=1}^{k} \alpha_{i} \frac{\beta_{i} s^{2}+\gamma_{i} s}{\beta_{i} s^{2}+\gamma_{i} s+\eta_{i}}
$$

and the operator $I_{\boldsymbol{3}}$ has been factored into

$$
I_{3}=E N(\mathbf{x})
$$

to yield a linear partial differential equation. The purpose of the factorization of $(7)$ is for the case of the beam or rod of varying cross section. For example, for a beam with varying cross section,

$$
N(x)=\frac{I(x)}{\rho A} \frac{\partial^{2}}{\partial^{2} x}
$$

where $x$ is the distance along the beam, $I(x)$ is the moment of area, $\rho$ is the material density, and $A$ it the cross sectional area. Note that when the force or displacement is represented by capital letters it denotes the Laplace transformed variable. 


\section{ANALYSIS}

Consider the solution of (5) by separation of variables/modal expansion with associated permissible boundary conditions. The solutions are then in the form

$$
w(\mathbf{x}, s)=\sum_{n=1}^{\infty} w_{n}(\mathbf{x}, s)=\sum_{n=1}^{\infty} a_{n}(s) \Phi_{n}(\mathbf{x})
$$

Substituting (9) into (5) yields

$$
\sum_{n=1}^{\infty}\left[\left(s^{2}+s d+E^{*}(s) L_{2} N(\mathbf{x})\right) A_{n}(s)\right] \Phi_{n}(\mathbf{x})-(s+d) w(\mathbf{x}, 0)-\dot{w}(\mathbf{x}, 0)=F(\mathbf{x}, s)
$$

If $\Phi_{n}(\mathbf{x})$ are chosen to be the eigenfunctions of the operator $L_{2} N(\mathbf{x})$ such that the boundary conditions are satisfied and

$$
L_{2} N(\mathbf{x}) \Phi_{n}=\lambda_{n} \Phi_{n}
$$

then (10) becomes

$$
\sum_{n=1}^{\infty}\left[\left(s^{2}+s d+E^{*}(s) \lambda_{n}\right) A_{n}(s)\right] \Phi_{n}(\mathbf{x})-(s+d) w(\mathbf{x}, 0)-\dot{w}(\mathbf{x}, 0)=F(\mathbf{x}, s)
$$

where $\lambda_{n}$ are the eigenvalues of the associated with the eigenfunctions $\Phi_{n}(\mathbf{x})$. Note that (11) is the usual modal assumption for the undamped equations of motion.

The functions are assumed to be orthonormal such that

$$
\int_{\Omega} \Phi_{n}(\mathbf{x}) \Phi_{m}(\mathbf{x}) d \Omega=\delta_{r m}
$$

where $\Omega$ is the spacial domain of the equation, i.e. length for a beam and area for a plate.

Multiplying (12) by $\Phi_{m}(\mathbf{x})$, integrating over the region $\Omega$ and applying (13) yields the $n$ temporal equations

$$
\left[\left(s^{2}+s d+E^{*}(s) \lambda_{n}\right) A_{n}(s)-(s+d) a_{n}(0)-a_{n}(0)\right]=f_{n}(s)
$$

where

$$
f_{n}(s)=\int_{\Omega} F(\mathbf{x}, s) \Phi_{n}(\mathbf{x}) d \Omega
$$

and

$$
a_{n}(0)=\int_{\mathbf{\Omega}} w(\mathbf{x}, 0) \Phi_{n}(\mathbf{x}) d \Omega \quad, \quad \dot{a}_{n}(0)=\int_{\Omega} \dot{w}(\mathbf{x}, 0) \Phi_{n}(\mathbf{x}) d \Omega
$$


The solution of the temporal equation is then

$$
A_{n}(s)=\frac{\left[f_{n}+(s+d) a_{n}(0)+\dot{a}_{n}(0)\right] \prod_{j=1}^{k} d_{j}}{\prod_{j=t}^{k} d_{j}\left(s^{2}+s d+E \lambda_{n}\right)+E \lambda_{n} \sum_{i=1}^{k}\left[\alpha_{i}\left(d_{i}-\eta_{i}\right) \prod_{\substack{j=1 \\ j \neq i}}^{k} d_{j}\right]}
$$

where (3) has been substituted into the solution and $d_{i}=\beta_{i} s^{2}+\gamma_{i} s+\eta_{i}$.

\section{EXAMPLE}

Consider a problem involving decay from initial conditions of a simply supported $1 \mathrm{~cm}$ thick $1.5 \mathrm{~m} \times 2 \mathrm{~m}$ plate given an assumed complex modulus. A complex modulus represented by a single expansion term has been arbitrarily chosen in order to obtain a simple solution. The parameters are as labeled.

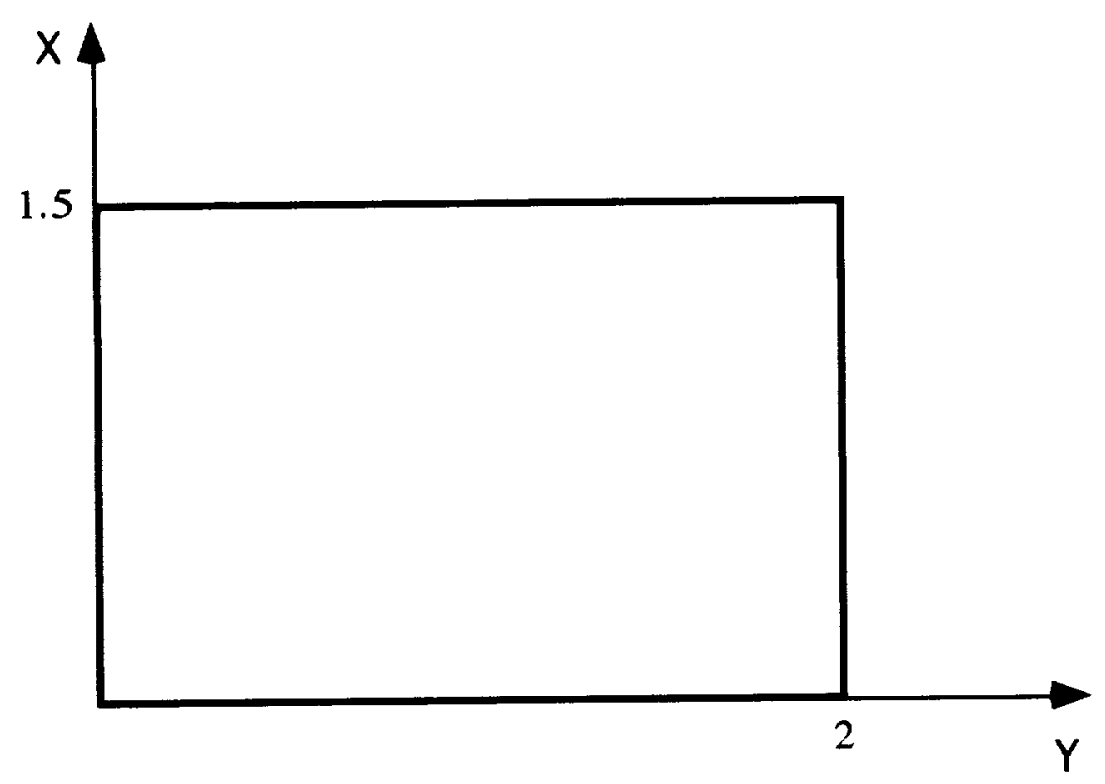

\section{Figure 1}

$$
\rho=2700 \frac{\mathrm{kg}}{\mathrm{m}^{3}}, E=6.89 \times 10^{10} \mathrm{~Pa}, v=.34, h=.01 \mathrm{~m}
$$

The viscoelastic properties are $\alpha=6, \beta=1, \gamma=1.5 \times 10^{4}, \mu=1.5 \times 10^{6}$.

The initial conditions are given as $w(x, y, 0)=.01 \sin \left(\frac{x \pi}{1.5}\right) \sin \left(\frac{y \pi}{2}\right)$, which is the first mode shape, with an amplitude of .01. The following time derivatives are also set: $\dot{w}(x, y, 0)=\ddot{w}(x, y, 0)=\ddot{w}(x, y, 0)=0$. The need for this will become apparent. The equation of motion for a plate is 


$$
s^{2} W(x, y, s)+\frac{D^{*}(s)}{\rho h} \nabla^{4} W(x, y, s)=\frac{F(x, y, s)}{\rho h}
$$

From eqns. (3) and (5),

$$
\frac{D(s)}{\rho h}=\frac{1}{\rho h} \frac{E^{*}(s) h^{3}}{12\left(1-v^{2}\right)}=240.5\left(1+.6 \frac{s^{2}+1.5 \times 10^{4} s}{s^{2}+1.5 \times 10^{4} s+1.5 \times 10^{6}}\right)
$$

where $\mathrm{m}$ and $\mathrm{n}$ are integers from 1 to $\infty$. The natural frequencies of an undamped simply supported plate are given by (See Reismann ${ }^{1}$ for instance)

$$
\Omega_{m n}=\sqrt{\operatorname{Re}(D(s) / \rho h)}\left[\left(\frac{m \pi}{1.5}\right)^{2}+\left(\frac{n \pi}{2}\right)^{2}\right]
$$

Using eqns. (E2) and (E3), $\Omega_{11}=124.14 \mathrm{rad} / \mathrm{sec}$, and $\mathrm{K}(124.14 \mathrm{i})=328.3(1+.217$ i). From eqn. (17)

$$
A(s)=\frac{s^{2}+1.5 \times 10^{4} s+1.5 \times 10^{6}}{s^{4}+1.5 \times 10^{4} s^{3}+1.5247 \times 10^{6} s^{2}+3.6986 \times 10^{8} s+2.3116 \times 10^{10}}
$$

which can be represented in state space form as

$$
\left[\begin{array}{l}
\dot{z}_{1} \\
\dot{z}_{2} \\
\dot{z}_{3} \\
\dot{z}_{4}
\end{array}\right]=\left[\begin{array}{cccc}
0 & 1 & 0 & 0 \\
0 & 0 & 1 & 0 \\
0 & 0 & 0 & 1 \\
-1.69 \times 10^{10} & -2.71 \times 10^{8} & -1.52 \times 10^{6} & -1.5 \times 10^{4}
\end{array}\right]\left[\begin{array}{l}
z_{1} \\
z_{2} \\
z_{3} \\
z_{4}
\end{array}\right]
$$

and

$$
a(t)=\left[\begin{array}{llll}
5.6 \times 10^{4} & 5.6 \times 10^{4} & 3.7 \times 10-2 & 0
\end{array}\right]\left[\begin{array}{l}
z_{1} \\
z_{2} \\
z_{3} \\
z_{4}
\end{array}\right]
$$

where the states $z$ are related to the displacement by equation (E6) and the initial conditions are $a(0)=.01$ and $\dot{a}(0)=\ddot{a}(0)=\dddot{a}(0)=0$.

From eqn. (E6), its time derivatives, and the initial conditions,

$$
\left[\left.\begin{array}{l}
a \\
\dot{a} \\
\ddot{a} \\
\ddot{a}
\end{array}\right|_{=0}=\left.\left[\begin{array}{cccc}
5.6 \times 10^{4} & 5.6 \times 10^{2} & 3.7 \times 10^{-2} & 0 \\
0 & 5.6 \times 10^{4} & 5.6 \times 10^{2} & 3.7 \times 10^{-2} \\
-6.3 \times 10^{8} & -1.0 \times 10^{7} & -6.7 \times 10^{2} & 0 \\
0 & -6.3 \times 10^{8} & -1.0 \times 10^{7} & -6.7 \times 10^{2}
\end{array}\right]\left[\begin{array}{c}
z_{1} \\
z_{2} \\
z_{3} \\
z_{4}
\end{array}\right]\right|_{-0}=\left[\begin{array}{c}
.01 \\
0 \\
0 \\
0
\end{array}\right],\right.
$$

the solution of which is 


$$
\left.\left[\begin{array}{l}
z_{1} \\
z_{2} \\
z_{3} \\
z_{4}
\end{array}\right]\right|_{-0}=\left[\begin{array}{c}
4.8 \times 10^{-7} \\
-1.6 \times 10^{-6} \\
-4.3 \times 10^{-1} \\
6.4 \times 10^{3}
\end{array}\right]
$$

The results of a digital simulation of the plate response are shown in figure 2 . The decay envelope for an equivalent viscous damped plate $(\xi=\eta / 2$ where $\eta$ is the loss factor of the material at $124.14 \mathrm{rad} / \mathrm{sec}$ ) is shown for comparison.

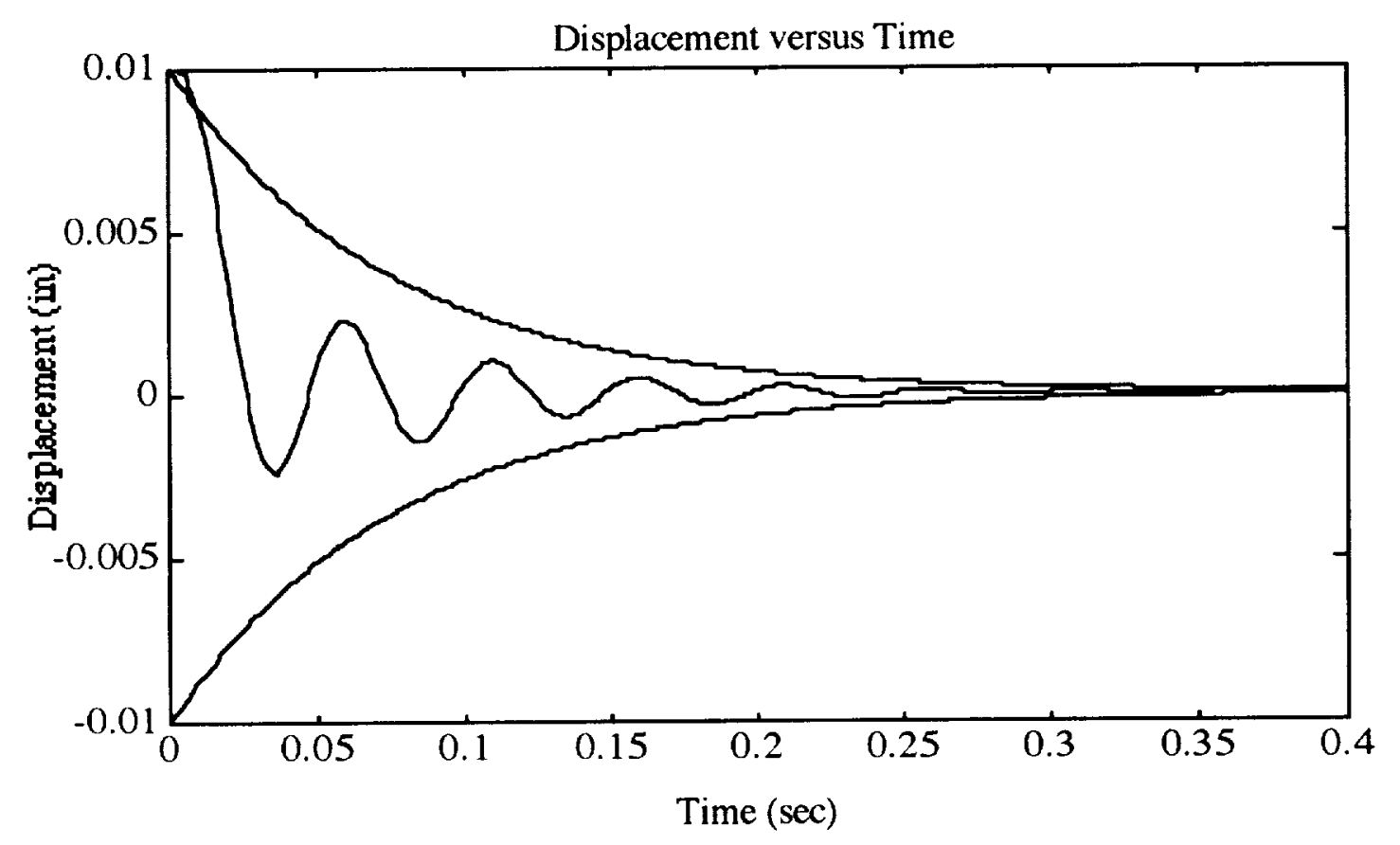

Figure 2

\section{DISCUSSION}

A method has been presented solving viscoelastic plate problems which accounts for frequency dependent modulus of elasticity. The solution yields the same orthogonal eigenfunctions/modes as classical plate theory while producing decaying temporal functions representing viscoelastic effects. This method provides a simple approximation for modelling viscoelastic plates. The solution reduces to the classical Sophie Germain solution when the hysteresis terms are dropped, as can be seen in equation (17) when $\alpha_{n}$ is set equal to zero. 


\section{ACKNOWLEDGEMENTS}

This work was supported by NASA Langley Research Center, Grant No. NGT50541, under the direction of Dr. Garnett Horner.

\section{REFERENCES}

1) Reismann, H., Elastic Plates, Theory and Application, John Wiley \& Sons, New York, 1988.

2) Soedel, W., Vibration of Shells and Plates, Marcel Dekker, Inc., New York, 1981.

3) Cudney, H.H. and Inman, D.J., "Determining Damping Mechanisms in a Composite Beam by Experimental Modal Analysis," International Journal of Analytical and Experimental Modal Analysis, Vol. 4, No. 4, Oct. 1989, pp. 138-143.

4) Banks, H.T., Fabiano, R.H., Wang, Y., Inman, D.J., and Cudney, H.H., "Spatial Versus Time Hysteresis in Damping Mechanisms," Proceedings of the 27th IEEE CDC, 1988, pp. 1674-1677.

5) Inman, D.J., "Vibration Analysis of Viscoelastic Beams by Separation of Variables and Model Analysis," Mechanics Research Communications, Vol. 16, Apr. 1989, pp. 213-218.

6) Slater, J.C. and Inman, D.J., "Transfer Function Modeling of Damping Mechanisms in Viscoelastic Plates," 32nd Structures, Structural Dynamics, and Materials Conference, Apr. 1991, pp. 2381-2383.

7) Christensen, R.M., Theory of Viscoelasticity: An Introduction, Academic Press, New York, 1982.

8) Banks, H.T. and Inman, D.J., “On Damping Mechanisms in Beams," No. \#89-3, University of Southern California, Sep. 1989.

9) Golla, D.F. and Hughes, P.C., "Dynamics of Viscoelastic Structures-a Time Domain Finite Element Formulation," ASME Journal of Applied Mechanics, Vol. 52, Dec. 1985, pp. 897-906. 
